OPEN ACCESS

Edited by: Mitali Dandekar,

Paras Cancer Centre, India

Reviewed by: loannis Vasileiadis,

Brighton and Sussex Medical School, United Kingdom

Zhihui Li,

Sichuan University, China

*Correspondence:

Zeyu Wu

wu.zeyu@hotmail.com

Yongchen Liu

avatarlara@163.com

${ }^{+}$These authors have contributed equally to this work and

share first authorship

Specialty section: This article was submitted to Head and Neck Cancer, a section of the journal

Frontiers in Oncology

Received: 21 June 2020 Accepted: 30 April 2021

Published: 19 May 2021

Citation:

Huang J, Song $M$, Shi H, Huang $Z$, Wang S, Yin Y, Huang Y, Du J,

Wang S, Liu Y and Wu Z (2021)

Predictive Factor of Large-Volume Central Lymph Node Metastasis in

Clinical No Papillary Thyroid Carcinoma Patients Underwent Total Thyroidectomy.

Front. Oncol. 11:574774. doi: 10.3389/fonc.2021.574774

\section{Predictive Factor of Large-Volume Central Lymph Node Metastasis in Clinical NO Papillary Thyroid Carcinoma Patients Underwent Total Thyroidectomy}

\author{
Jianhao Huang ${ }^{1,2 \dagger}$, Muye Song ${ }^{1,3 \dagger}$, Hongyan Shi ${ }^{1,4 \dagger}$, Ziyang Huang ${ }^{1,2}$, Shujie Wang ${ }^{1,4}$, \\ Ying Yin ${ }^{1}$, Yijie Huang ${ }^{1}$, Jialin Du ${ }^{1}$, Sanming Wang ${ }^{1}$, Yongchen Liu $^{1 *}$ and Zeyu Wu ${ }^{1 *}$ \\ ${ }^{1}$ Department of General Surgery, Guangdong Provincial People's Hospital, Guangdong Academy of Medical Sciences, \\ Guangzhou, China, ${ }^{2}$ Shantou University Medical College, Shantou University, Shantou, China, ${ }^{3}$ School of Medicine, South \\ China University of Technology, Guangzhou, China, ${ }^{4}$ The Second School of Clinical Medicine, Southern Medical University, \\ Guangzhou, China
}

Large-volume central lymph node metastasis (large-volume CLNM) is associated with high recurrence rate in papillary thyroid carcinoma (PTC) patients. However, sensitivity in investigating large-volume CLNM on preoperative ultrasonography (US) is not high. The aim of this study is to investigate the clinical factors associated with large-volume CLNM in clinical NO PTC patients. We reviewed 976 PTC patients undergoing total thyroidectomy with central lymph node dissection during 2017 to 2019. The rate of large-volume LNM was $4.1 \%$ (40 of 967 patients). Multivariate analysis showed that male gender and young age (age $<45$ years old) were independent risk factors for large-volume CLNM with odds ratios $[(\mathrm{OR}), 95 \%$ confidence interval $(\mathrm{Cl})]$ of 2.034 (1.015-4.073) and 2.997 (1.3066.876), respectively. In papillary thyroid microcarcinoma (PTMC), capsule invasion was associated with large-volume CLNM with OR (95\% Cl) of 2.845 (1.110-7.288). In conventional papillary thyroid cancer (CPTC), tumor diameter $(>2 \mathrm{~cm})$ was associated with large-volume CLNM, with OR (95\% Cl) 3.757 (1.061-13.310), by multivariate analysis. In ROC curve analysis on the diameter of the CPTC tumor, the Area Under Curve $(A \cup C)=0.682(p=0.013)$, the best cut-off point was selected as $2.0 \mathrm{~cm}$. In conclusion, male gender and young age were predictors for large-volume CLNM of cNO PTC. cNO PTMC patient with capsule invasion and cNO CPTC patient with tumor diameter $>2 \mathrm{~cm}$ were correlated with large-volume CLNM. Total thyroidectomy with central lymph node dissection may be a favorable primary treatment option for those patients.

Keywords: central lymph node metastasis, total thyroidectomy, tumor diameter, conventional papillary thyroid cancer, capsule invasion 


\section{INTRODUCTION}

Papillary thyroid cancer (PTC) is the most common type of differentiated thyroid cancer, which account for $85 \%-90 \%$ of all cases (1). The incidence of PTC has been rapidly increasing globally because of early detection (2). According to the World Health Organization (WHO), PTMC is defined as PTC with maximum diameter of $10 \mathrm{~mm}$. Those with maximum diameter of more than $10 \mathrm{~mm}$ is called CPTC (3).

PTC has a high metastasis rate especially central lymph node metastasis (CLNM), approximately $30 \%-80 \%$ of PTC patients have CLNM (4). It has been reported that $30 \%$ to $65 \%$ of patients with cN0 PTC are detected CLNM (5). The recurrence rate of patients with CLNM mainly depends on the number of involved lymph node and the size of the largest lymph node (6). Patients with large-volume lymph node metastasis ( $>5$ involved lymph nodes) can significantly increase the risk of recurrence during long-time survival, associated with $20 \%$ recurrence rates, while small-volume metastasis ( $\leq 5$ involved lymph nodes) accounts for $5 \%$ recurrence rates $(7)$.

In the previous study, young age and male sex have been highlighted with large-volume CLNM in PTMC patient, suggesting surgery may be the primary option for young male patients (8). PTMC with multifocality, tumor diameter $>0.5 \mathrm{~cm}$ and extrathyroidal extension (ETE) also tends to have largevolume CLNM $(9,10)$. However, the clinicopathologic factors associated with large-volume CLNM in CPTC are not wellestablished (11). Sensitivity of ultrasound examination for central neck lymph node metastasis was not high (12). Therefore, identifying risk factors for large-volume CLNM in clinical N0 PTC patients might be helpful in deciding on a management strategy in PTC patients.

\section{MATERIALS AND METHODS}

\section{Study Population}

A total of 976 PTC patients who received primary total thyroidectomy with central lymph node dissection treatment in the Department of General Surgery at Guangdong General Hospital between October 2017 to September 2019 were reviewed retrospectively in this study. The study was approved by Research Ethics Committee of Guangdong Provincial People's Hospital, Guangdong Academy of Medical Sciences. All patients gave their informed consent to the collection of data according to the local ethic committee indications. Ultrasound examination (US) was routinely performed to assess the thyroid and lymph node status in all these patients. Computed tomography scan (CT) and positron emission tomography-computed tomography (PET-CT) were used only in some patients as needed. Patients were diagnosed PTC with LNM by pathological examination in the Pathology Department. LNM was defined as preoperatively $\mathrm{cN} 1$ with following signs in preoperative ultrasound examination: the ration of transverse/long diameter in cervical lymph node $>0.5$, blurred corticomedullary boundary, vanished medulla structure, microcalcification or cystic changes (13).
Patients were excluded if they exhibited the following criteria (I) pathologic-confirmed not PTC; (II) met preoperatively $\mathrm{cN} 1$ diagnostic criteria; (III) received prior surgery or radiotherapy of the neck. (IV) confirmed distant metastasis or gross extrathyroidal extension.

After excluding 612 patients with suspected CLNM preoperatively, 364 patients were involved. Gender, age, ultrasound features and pathologic characteristics are recorded. Patients were divided into two groups by age: (I) age $<45$ years old; (II)age $\geq 45$ years old. In cases of multifocal PTCs, the diameter of the largest tumor was used. ETE only accounted for microscopic extension of tumors. The diagnosis of autoimmune thyroid disease and nodule goiter was determined by general pathological examination.

\section{Statistical Analysis}

Statistical analysis was performed with IBM SPSS statistics 26.0 software. The relationships between large-volume LNM and clinicopathologic characteristics were investigated by the $\mathrm{X}^{2}$ test or one-way ANOVA to test univariate analyses. The multivariate analysis was performed on the variables that achieved $\mathrm{P}<0.05$ in the univariate analysis and predictive factors for large-volume LNM were tested by the logistic regression analysis and the data was presented as the mean \pm SD. $\mathrm{P}$ values $<0.05$ (two sided) were considered statistically significant.

\section{RESULTS}

\section{Clinicopathological Characteristics of the Study Patients}

The clinicopathological characteristics of the 364 study patients according to CLNM status are listed in Table 1. Among these, 324 (89.0\%) had small-volume CLNM, and 40 (11.0\%) had largevolume CLNM. The mean age was $41.02 \pm 11.324$. The numbers of patients were $220(60.4 \%)$ and $144(39.6 \%)$ in Groups I-II, respectively. 235 patients were female (64.6\%), and 35.4\% (129 patients) were male. 232 (63.7\%) patients had PTMC, while 132 $(36.3 \%)$ patients had tumors larger than $1 \mathrm{~cm}$. With the pathology examination, a total of $66(18.1 \%)$ patients were diagnosed with Hashimoto's thyroiditis and 93 (25.5\%) patients had nodular goiter. 87 (23.9\%) patients had capsule invasion and $56(15.3 \%)$ patients had ETE.

\section{Risk Factors for Large-Volume CLNM in PTC}

In univariate analysis, young age ( $<45$ years), male, multifocality, nodule goiter and ETE were significantly associated with a high prevalence of large-volume CLNM (Table 1). However, bilateral tumors, marked hypoechoicity, irregular margin and non-welldefined shape were not correlated with the large-volume CLNM. In multivariate analysis, young age [odds ratio (OR): 2.034, 95\% confidential interval (CI): 1.015-4.073, $\mathrm{P}=0.045$ ] and male (OR: 2.997, 95\% CI: 1.306-6.876, $\mathrm{P}=0.010$ ) were still significant predictive factors for large-volume CLNM (Table 2). 
TABLE 1 | Clinicopathological characteristics and univariate analysis of PTC patients $(n=364)$.

\begin{tabular}{|c|c|c|c|}
\hline & Small-volume $(n=324)$ & Large-volume $(n=40)$ & p-value \\
\hline Gender & & & $0.017^{\star}$ \\
\hline Male & 108 & 21 & \\
\hline Female & 216 & 19 & \\
\hline Age & & & $0.046^{*}$ \\
\hline$<45$ & 190 & 30 & \\
\hline$\geq 45$ & 134 & 10 & \\
\hline Bilateral tumors & & & 0.094 \\
\hline Bilateral & 76 & 14 & \\
\hline Unilateral & 248 & 26 & \\
\hline Multifocal tumor & & & 0.754 \\
\hline Multifocal & 103 & 18 & \\
\hline single & 221 & 22 & \\
\hline Hashimoto's thyroiditis & & & 0.157 \\
\hline Yes & 62 & 4 & \\
\hline No & 262 & 36 & \\
\hline \multicolumn{4}{|l|}{ Nodule } \\
\hline Goiter & & & $0.049^{*}$ \\
\hline Yes & 87 & 5 & \\
\hline No & 237 & 35 & \\
\hline Marked Hypoechoic & & & 0.745 \\
\hline Yes & 266 & 32 & \\
\hline No & 58 & 8 & \\
\hline Margin & & & 0.207 \\
\hline Irregular & 276 & 31 & \\
\hline Regular & 48 & 9 & \\
\hline Shape & & & 0.167 \\
\hline Well-defined & 46 & 9 & \\
\hline Non-well-defined & 278 & 31 & \\
\hline Capsule Invasion & & & $0.003^{*}$ \\
\hline Yes & 70 & 17 & \\
\hline No & 254 & 23 & \\
\hline $\mathrm{ETE}^{\star \star}$ & & & $0.001^{*}$ \\
\hline Yes & 43 & 13 & \\
\hline No & 281 & 27 & \\
\hline
\end{tabular}

*statistically significance; **extrathyroidal extension.

\section{Risk Factors for Large-Volume CLNM in PTMC and CPTC}

Divided by the maximum diameter of the tumor, clinicopathologic characteristic were tested in PTMC patients and CPTC patients, respectively. In 232 PTMC patients, male, multifocality, nodule goiter and capsule invasion were significantly correlated with large-volume CLNM (Table 3). Age and ETE were not correlated with the large-volume CLNM. In multivariate analysis, shown in Table 4, only capsule invasion (OR: 2.845, 95\% CI: $1.110-7.288, \mathrm{P}=0.029)$ was still significant predictive factors for large-volume CLNM. In 132 CPTC patients, Hashimoto's thyroiditis, marked hypoechoicity, irregular margin and tumor diameter $(>2 \mathrm{~cm})$ were significantly correlated with large-volume CLNM (Table 5). Age and gender were not correlated with the large-volume CLNM. In multivariate analysis, shown in Table 6, tumor diameter ( $>2 \mathrm{~cm})$ (OR: $3.757,95 \%$ CI: 1.061-13.310, $\mathrm{P}=0.04)$ was significant predictive factors for large-volume CLNM. The high sensitivities and low falsenegative rates (1- specificity) associated with the tumor diameter were identified via ROC curve analysis, as depicted in Figure $\mathbf{1}$. The Area Under Curve (AUC) $=0.682(\mathrm{p}=0.013)$, the best cut-off point was selected as $2.0 \mathrm{~cm}$, which meant that CPTC patients with tumor diameter higher than $2.0 \mathrm{~cm}$ had more likelihood to have large-volume CLNM.

TABLE 2 | Multivariate analysis of the risk factors of PTC.

\begin{tabular}{lcr}
\hline & Sig. & OR (95\% Cl) \\
\hline Gender & 0.045 & $2.034(1.015-4.073)$ \\
Age & 0.010 & $2.997(1.306-6.876)$ \\
Capsule Invasion & 0.284 & $0.561(0.194-1.616)$ \\
ETE $^{*}$ & 0.136 & $0.408(0.125-1.326)$ \\
Nodule goiter & 0.111 & $2.254(0.829-6.130)$ \\
\hline
\end{tabular}

*extrathyroidal extension. 
TABLE 3 | Clinicopathological characteristics and univariate analysis of PTMC patients ( $\mathrm{n}=232$ ).

\begin{tabular}{|c|c|c|c|}
\hline & Small-volume $(n=210)$ & Large-volume $(n=22)$ & p-value \\
\hline Gender & & & $0.028^{*}$ \\
\hline Male & 74 & 13 & \\
\hline Female & 136 & 9 & \\
\hline Age & & & 0.184 \\
\hline$<45$ & 122 & 16 & \\
\hline$\geq 45$ & 88 & 6 & \\
\hline Bilateral tumors & & & 0.292 \\
\hline Bilateral & 46 & 7 & \\
\hline Unilateral & 164 & 15 & \\
\hline Multifocal tumor & & & $0.043^{*}$ \\
\hline Multifocal & 61 & 11 & \\
\hline single & 149 & 11 & \\
\hline Hashimoto's thyroiditis & & & 0.964 \\
\hline Yes & 39 & 4 & \\
\hline No & 171 & 18 & \\
\hline Nodule & & & 0.226 \\
\hline \multicolumn{4}{|l|}{ Goiter } \\
\hline Yes & 53 & 3 & \\
\hline No & 157 & 19 & \\
\hline Margin & & & 0.748 \\
\hline Irregular & 179 & 20 & \\
\hline Regular & 31 & 2 & \\
\hline Shape & & & 1.00 \\
\hline Well-defined & 30 & 3 & \\
\hline Non-well-defined & 180 & 19 & \\
\hline Marked Hypoechoic & & & 0.115 \\
\hline Yes & 173 & 21 & \\
\hline No & 37 & 1 & \\
\hline Invasion & & & $0.004^{*}$ \\
\hline Yes & 40 & 12 & \\
\hline No & 170 & 10 & \\
\hline ETE $^{\star \star}$ & & & 0.079 \\
\hline Yes & 28 & 6 & \\
\hline No & 182 & 16 & \\
\hline Size of tumors & & & 0.916 \\
\hline$>0.5$ & 136 & 14 & \\
\hline$\leq 0.5$ & 74 & 8 & \\
\hline
\end{tabular}

*statistically significance; **extrathyroidal extension.

TABLE 4 | Multivariate analysis of the risk factors of PTMC.

\begin{tabular}{|c|c|c|}
\hline & Sig. & OR (95\% Cl) \\
\hline Gender & 0.123 & $2.080(0.819-5.281)$ \\
\hline Multifocality & 0.121 & $2.064(0.826-5.160)$ \\
\hline Capsule Invasion & 0.029 & $2.845(1.110-7.288)$ \\
\hline
\end{tabular}

\section{DISCUSSION}

Previous studies have demonstrated that male sex, age, multifocality, tumor diameter $>0.5 \mathrm{~cm}$ and ETE were independent risk factors for LNM in PTMC patients (8-10). In this study, univariate and multivariate analyses revealed that age $<45$ years and male gender are independent predictive factors for large-volume CLNM in cN0 PTC patients. In addition, capsule invasion is independent predictive factors for largevolume CLNM in cN0 PTMC, while tumor diameter $>2 \mathrm{~cm}$ predicts large-volume CLNM in in CNO CPTC.

Age is regarded as the most important prognostic factors for thyroid cancer. 45 years is widely adopted as clinically important prognostic marker in PTC patients. Our study demonstrates that the rate of large-volume CLNM was higher in patients $<45$ years than that $\geq 45$ years $(13.6 \%$ vs. $6.9 \%, \mathrm{P}=0.046)$. Multivariate analysis shows age $<45$ years was independent predictor of largevolume CLNM inpatients with cN0 PTC. Similarly, previous studies have reported that CLNM was more likely to appear in PTC patients with age $<45$ years, which may be due to rapid tumor diameter growth in young patient during surveillance period $(6,14,15)$. These results indicate that much careful preoperative assessment of the lymph node status must be followed in young patients.

Male gender has been identified as a risk factor for thyroid carcinoma (16). Similarly, recent studies have revealed that men 
TABLE 5 | Clinicopathological characteristics and univariate analysis of CPTC patients ( $n=132)$.

\begin{tabular}{|c|c|c|c|}
\hline & Small-volume $(n=114)$ & Large-volume $(n=18)$ & p-value \\
\hline Gender & & & 0.216 \\
\hline Male & 34 & 8 & \\
\hline Female & 80 & 10 & \\
\hline Age & & & 0.141 \\
\hline$<45$ & 68 & 14 & \\
\hline$\geq 45$ & 46 & 4 & \\
\hline Bilateral tumors & & & 0.270 \\
\hline Bilateral & 30 & 7 & \\
\hline Unilateral & 84 & 11 & \\
\hline Multifocal tumor & & & 0.754 \\
\hline Multifocal & 40 & 7 & \\
\hline single & 74 & 11 & \\
\hline Hashimoto's thyroiditis & & & $0.042^{*}$ \\
\hline Yes & 19 & 0 & \\
\hline No & 91 & 18 & \\
\hline \multicolumn{4}{|l|}{ Nodule } \\
\hline Goiter & & & 0.406 \\
\hline Yes & 34 & 16 & \\
\hline No & 80 & 2 & \\
\hline Marked Hypoechoic & & & $0.048^{*}$ \\
\hline Yes & 93 & 11 & \\
\hline No & 12 & 7 & \\
\hline Margin & & & $0.022^{*}$ \\
\hline Irregular & 97 & 11 & \\
\hline Regular & 17 & 7 & \\
\hline Shape & & & 0.08 \\
\hline Well-defined & 16 & 6 & \\
\hline Non-well-defined & 98 & 12 & \\
\hline Capsule Invasion & & & 0.27 \\
\hline Yes & 30 & 7 & \\
\hline No & 84 & 11 & \\
\hline $\mathrm{ETE}^{\star \star}$ & & & $0.013^{\star}$ \\
\hline Yes & 15 & 7 & \\
\hline No & 99 & 11 & \\
\hline Size of tumors & & & $0.005^{\star}$ \\
\hline$>2 \mathrm{~cm}$ & 16 & 8 & \\
\hline$\leq 2 \mathrm{~cm}$ & 98 & 10 & \\
\hline
\end{tabular}

*statistically significance; **extrathyroidal extension.

TABLE 6 | Multivariate analysis of the risk factors of CPTC.

\begin{tabular}{|c|c|c|}
\hline & Sig. & OR (95\% Cl) \\
\hline Hashimoto's & 0.998 & 0.00 \\
\hline \multicolumn{3}{|l|}{ Thyroiditis } \\
\hline Margin & 0.596 & $0.649(0.131-3.219)$ \\
\hline Shape & 0.419 & $0.533(0.116-2.452)$ \\
\hline Diameter & 0.040 & $3.757(1.061-13.310)$ \\
\hline ETE $^{\star}$ & 0.072 & $3.194(0.900-11.332)$ \\
\hline
\end{tabular}

*extrathyroidal extension.

exhibited aggressive behavior and poorer prognosis than women among PTC patients (17). Among PTC patients who did not undergo prophylactic central neck dissection, male gender is a risk factor for further recurrence (18). This study also showed male gender as the predictive factor for large-volume CLNM in cN0 PTC. Therefore, male patient with cN0 PTC should be evaluated carefully and complete central neck dissection may be favorable.

With the increased diagnosis of PTC, a growing number of patients with PTMC have been detected. PTMC appears to account for the majority of the increased incidence of thyroid cancer (19). Capsule invasion is considered as aggressive behavior of PTMC, which is correlated with CLNM, even with lymph nodes posterior to right recurrent laryngeal nerve metastasis (LN-prRLN) $(14,20)$. In PTMC, previous studies did not demonstrate the clear relationship between capsule invasion and large- volume metastasis. A novel finding in our study is that capsule invasion is more likely to present with largevolume metastasis. ( $\mathrm{P}=0.029, \mathrm{OR}=2.845$, with $95 \% \mathrm{CI}$ : ranged from 1.110-7.288). The incidence of large-volume CLNM in 


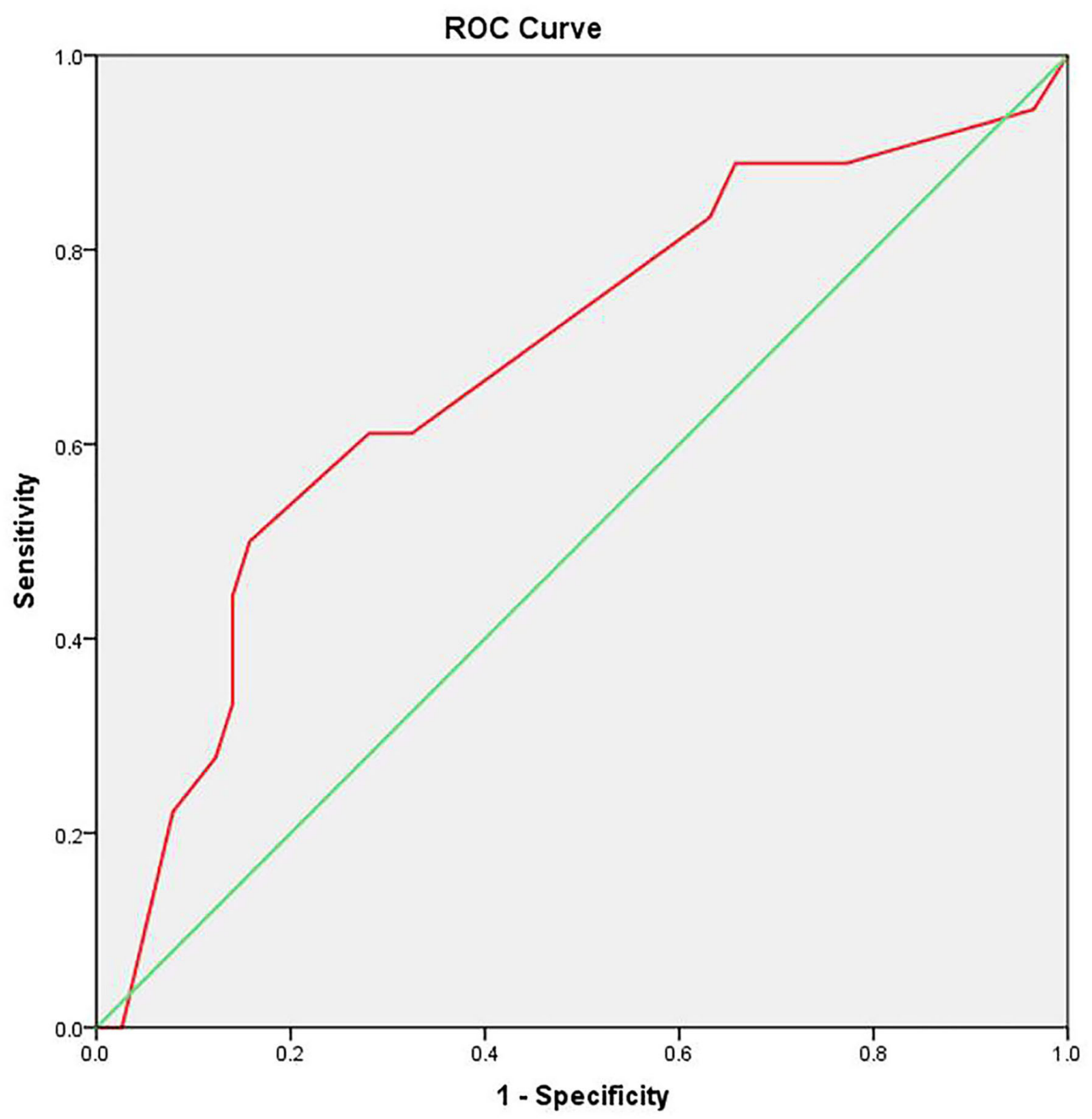

$p=0.013$ AUC $=0.682$ best cut-off point $=2.0 \mathrm{~cm}$

FIGURE 1 | ROC curve analysis of the cutoff point for diameter of the CPTC tumor.

capsule invasion and non-capsule invasion were $23 \%$ vs. $5.6 \%$, with a $\mathrm{P}$ value $<0.05$ in multivariate analysis. It might be expected that the PTMC with capsule invasion more likely showed extrathyroidal metastasis potential to lymph node and distant organs, which significantly accounted for the high incidence of large-volume CLNM and high recurrence rate.

Tumor size is considered as an important factor in TNM staging for PTC, and larger tumors tend to be more aggressive $(21,22)$. Recent studies reported that tumor diameter $>0.5 \mathrm{~cm}$ was independent risk factors for large-volume CLNM in cN0 PTMC $(8,9)$. However, Shen et al. (10) demonstrated that the tumor diameter $>0.5 \mathrm{~cm}$ was not significantly different between the large-volume CLNM group and non- large-volume CLNM volume group. We were also unable to investigate the relationship between tumor diameter and large volume metastasis in cN0 PTMC. However, in CPTC, we found that tumor diameter higher than $2 \mathrm{~cm}$ is associated with large-volume CLNM. Ito et al. (23) and Ma et al. (14) reported that tumor diameter of $>2 \mathrm{~cm}$ is the strongest predictor of CLNM and lymph node recurrence in PTC. Compared with tumor diameter $<2 \mathrm{~cm}$, tumor diameter $>2 \mathrm{~cm}$ was correlated with the five times higher risk of recurrence in PTC patients aged $\geq 55$ years old (24). ROC curve analysis was used to determine the cutoff point of tumor size for predicting large-volume metastasis and found that tumor diameter higher than $2 \mathrm{~cm}$ was the strongest predictor of largevolume CLNM in CN0 CPTC. Therefore, tumor diameter higher than $2 \mathrm{~cm}$ should be evaluated carefully for possible large-volume CLNM of PTC. In addition, careful prophylactic central node dissection should be recommended for N0 CPTC with a large tumor diameter (tumor diameter $>2 \mathrm{~cm}$ ). 
In our study, ultrasound features are analyzed to study the correlation with large-volume CLNM in PTMC and CPTC. However, no predictive factor is established. The possible reason is that ultrasonography in investigating CLNM is not sensitive and ultrasonography diagnosis is subjective, mainly relying on doctor's clinical experience $(25,26)$. In presence studies, radiomics and deep machinery learning are gradually conducted to investigate the CLNM. The highest sensitivity has reach 0.858 in the existing predictive models of CLNM, which is significantly higher than the current situation (lower than 0.5 for predicting CLNM) (27). It may provide more dependable evidence for performing central lymph node dissection.

However, the present study has several limitations. The risk factors we identified were based on our retrospective design and the relatively small number of patients with large-volume CLNM, and thus may need further exploration. In addition, our study has no information about follow-up evaluation of the patients for potential development of recurrence and future distant metastases.

\section{CONCLUSION}

In conclusion, age $<45$ years and male gender are independent predictors of large-volume CLNM metastasis in patients with cN0 PTC. Total thyroidectomy with central neck dissection may be a favorable option for surgeons when treating young male patients with $\mathrm{cN} 0$ PTC. Besides, $\mathrm{cN} 0$ PTMC patient with capsule invasion and $\mathrm{CN} 0$ CPTC patient with tumor diameter $>2 \mathrm{~cm}$ have higher prevalence of large-volume CLNM. Therefore, these factors should be considered by the surgeons when evaluating the risk of large-volume CLNM of PTC. Follow-up visits for post-operative patients with these pathologic characteristics should be regarded.

\section{REFERENCES}

1. Siegel RL, Miller KD, Jemal A. Cancer Statistics, 2019. CA Cancer J Clin (2019) 69(1):7-34. doi: 10.3322/caac.21551

2. Vigneri R, Malandrino P, Vigneri P. The Changing Epidemiology of Thyroid Cancer: Why is Incidence Increasing? Curr Opin Oncol (2015) 27(1):1-7. doi: $10.1097 /$ cco.0000000000000148

3. Thompson L. World Health Organization Classification of Tumours: Pathology and Genetics of Head and Neck Tumours. Ear Nose Throat J (2006) 85:74. doi: 10.1177/014556130608500201

4. Lee YM, Sung TY, Kim WB, Chung KW, Yoon JH, Hong SJ. Risk Factors for Recurrence in Patients With Papillary Thyroid Carcinoma Undergoing Modified Radical Neck Dissection. Br J Surg (2016) 103(8):1020-5. doi: 10.1002/bjs.10144

5. Wada N, Duh QY, Sugino K, Iwasaki H, Kameyama K, Mimura T, et al. Lymph Node Metastasis From 259 Papillary Thyroid Microcarcinomas: Frequency, Pattern of Occurrence and Recurrence, and Optimal Strategy for Neck Dissection. Ann Surg (2003) 237(3):399-407. doi: 10.1097/ 01.Sla.0000055273.58908.19

6. Adam MA, Pura J, Goffredo P, Dinan MA, Reed SD, Scheri RP, et al. Presence and Number of Lymph Node Metastases are Associated With Compromised Survival for Patients Younger Than Age 45 Years With Papillary Thyroid Cancer. J Clin Oncol (2015) 33(21):2370-5. doi: 10.1200/jco.2014.59.8391

\section{DATA AVAILABILITY STATEMENT}

The raw data supporting the conclusions of this article will be made available by the authors, without undue reservation.

\section{ETHICS STATEMENT}

The study was approved by Research Ethics Committee of Guangdong Provincial People's Hospital, Guangdong Academy of Medical Sciences. All patients gave their informed consent to the collection of data according to the local ethics committee's indications.

\section{AUTHOR CONTRIBUTIONS}

$\mathrm{JH}, \mathrm{MS}$, and HS designed this study. JH, MS, HS, ZH, and SJW collected the data. JH, MS, and HS analyzed the data. All authors contributed to the article and approved the submitted version.

\section{FUNDING}

This work was supported by the Natural Science Foundation of Guangdong Province (No. 2020A1515010127), Scientific Research Staring Foundation for the Returned Overseas from Guangdong Provincial People's Hospital (No. 2017x02) and Guangdong Provincial People's Hospital Scientific Foundation for Distinguished Young Scholars of Guangdong Province (No. KJ012019441).

7. Haugen BR, Alexander EK, Bible KC, Doherty GM, Mandel SJ, Nikiforov YE, et al. 2015 American Thyroid Association Management Guidelines for Adult Patients With Thyroid Nodules and Differentiated Thyroid Cancer: The American Thyroid Association Guidelines Task Force on Thyroid Nodules and Differentiated Thyroid Cancer. Thyroid (2016) 26(1):1-133. doi: 10.1089/thy.2015.0020

8. Oh HS, Park S, Kim M, Kwon H, Song E, Sung TY, et al. Young Age and Male Sex Are Predictors of Large-Volume Central Neck Lymph Node Metastasis in Clinical N0 Papillary Thyroid Microcarcinomas. Thyroid (2017) 27(10):128590. doi: 10.1089/thy.2017.0250

9. Liu C, Liu Y, Zhang L, Dong Y, Hu S, Xia Y, et al. Risk Factors for HighVolume Lymph Node Metastases in cN0 Papillary Thyroid Microcarcinoma. Gland Surg (2019) 8(5):550-6. doi: 10.21037/gs.2019.10.04

10. Shen G, Ma H, Huang R, Kuang A. Predicting Large-Volume Lymph Node Metastasis in the Clinically Node-Negative Papillary Thyroid Microcarcinoma: A Retrospective Study. Nucl Med Commun (2020) 41(1):5-10. doi: 10.1097/ mnm.0000000000001119

11. Wang JB, Sun YY, Shi LH, Xie L. Predictive Factors for non-Small-Volume Central Lymph Node Metastases (More Than 5 or $\geq 2 \mathrm{Mm}$ ) in Clinically Node-Negative Papillary Thyroid Carcinoma. Med (Baltimore) (2019) 98(1): e14028. doi: 10.1097/md.0000000000014028

12. Hwang HS, Orloff LA. Efficacy of Preoperative Neck Ultrasound in the Detection of Cervical Lymph Node Metastasis From Thyroid Cancer. Laryngoscope (2011) 121(3):487-91. doi: 10.1002/lary.21227 
13. Podnos YD, Smith D, Wagman LD, Ellenhorn JD. The Implication of Lymph Node Metastasis on Survival in Patients With Well-Differentiated Thyroid Cancer. Am Surg (2005) 71(9):731-4. doi: 10.1177/000313480507100907

14. Ma B, Wang Y, Yang S, Ji Q. Predictive Factors for Central Lymph Node Metastasis in Patients With cN0 Papillary Thyroid Carcinoma: A Systematic Review and Meta-Analysis. Int J Surg (2016) 28:153-61. doi: 10.1016/ j.ijsu.2016.02.093

15. Jiang LH, Yin KX, Wen QL, Chen C, Ge MH, Tan Z. Predictive Risk-scoring Model For Central Lymph Node Metastasis and Predictors of Recurrence in Papillary Thyroid Carcinoma. Sci Rep (2020) 10(1):710. doi: 10.1038/s41598019-55991-1

16. Lee YH, Lee YM, Sung TY, Yoon JH, Song DE, Kim TY, et al. Is Male Gender a Prognostic Factor for Papillary Thyroid Microcarcinoma? Ann Surg Oncol (2017) 24(7):1958-64. doi: 10.1245/s10434-017-5788-4

17. Ding J, Wu W, Fang J, Zhao J, Jiang L. Male Sex is Associated With Aggressive Behaviour and Poor Prognosis in Chinese Papillary Thyroid Carcinoma. Sci Rep (2020) 10(1):4141. doi: 10.1038/s41598-020-60199-9

18. Nixon IJ, Wang LY, Ganly I, Patel SG, Morris LG, Migliacci JC, et al. Outcomes for Patients With Papillary Thyroid Cancer Who do Not Undergo Prophylactic Central Neck Dissection. Br J Surg (2016) 103 (3):218-25. doi: 10.1002/bjs.10036

19. Leboulleux S, Tuttle RM, Pacini F, Schlumberger M. Papillary Thyroid Microcarcinoma: Time to Shift From Surgery to Active Surveillance? Lancet Diabetes Endocrinol (2016) 4(11):933-42. doi: 10.1016/s2213-8587(16)30180-2

20. Li C, Xiang J, Wang Y. Risk Factors for Predicting Lymph Nodes Posterior to Right Recurrent Laryngeal Nerve (LN-Prrln) Metastasis in Thyroid Papillary Carcinoma: A Meta-Analysis. Int J Endocrinol (2019) 2019:7064328. doi: 10.1155/2019/7064328

21. Kim M, Kim HI, Jeon MJ, Kim HK, Kim EH, Yi HS, et al. Eighth Edition of Tumor-Node-Metastasis Staging System Improve Survival Predictability for Papillary, But Not Follicular Thyroid Carcinoma: A Multicenter Cohort Study. Oral Oncol (2018) 87:97-103. doi: 10.1016/j.oraloncology.2018.10.029

22. Tuttle RM, Haugen B, Perrier ND. Updated American Joint Committee on Cancer/Tumor-Node-Metastasis Staging System for Differentiated and
Anaplastic Thyroid Cancer (Eighth Edition): What Changed and Why? Thyroid (2017) 27(6):751-6. doi: 10.1089/thy.2017.0102

23. Ito Y, Fukushima M, Higashiyama T, Kihara M, Takamura Y, Kobayashi K, et al. Tumor Size is the Strongest Predictor of Microscopic Lymph Node Metastasis and Lymph Node Recurrence of N0 Papillary Thyroid Carcinoma. Endocr J (2013) 60(1):113-7. doi: 10.1507/endocrj.ej12-0311

24. Tam S, Amit M, Boonsripitayanon M, Busaidy NL, Cabanillas ME, Waguespack SG, et al. Effect of Tumor Size and Minimal Extrathyroidal Extension in Patients With Differentiated Thyroid Cancer. Thyroid (2018) 28 (8):982-90. doi: 10.1089/thy.2017.0513

25. Chen J, Li XL, Zhao CK, Wang D, Wang Q, Li MX, et al. Conventional Ultrasound, Immunohistochemical Factors and BRAF(V600E) Mutation in Predicting Central Cervical Lymph Node Metastasis of Papillary Thyroid Carcinoma. Ultrasound Med Biol (2018) 44(11):2296-306. doi: 10.1016/ j.ultrasmedbio.2018.06.020

26. Huang XP, Ye TT, Zhang L, Liu RF, Lai XJ, Wang L, et al. Sonographic Features of Papillary Thyroid Microcarcinoma Predicting High-Volume Central Neck Lymph Node Metastasis. Surg Oncol (2018) 27(2):172-6. doi: 10.1016/j.suronc.2018.03.004

27. Zhou SC, Liu TT, Zhou J, Huang YX, Guo Y, Yu JH, et al. An Ultrasound Radiomics Nomogram for Preoperative Prediction of Central Neck Lymph Node Metastasis in Papillary Thyroid Carcinoma. Front Oncol (2020) 10:1591. doi: $10.3389 /$ fonc. 2020.01591

Conflict of Interest: The authors declare that the research was conducted in the absence of any commercial or financial relationships that could be construed as a potential conflict of interest.

Copyright (C) 2021 Huang, Song, Shi, Huang, Wang, Yin, Huang, Du, Wang, Liu and Wu. This is an open-access article distributed under the terms of the Creative Commons Attribution License (CC BY). The use, distribution or reproduction in other forums is permitted, provided the original author(s) and the copyright owner(s) are credited and that the original publication in this journal is cited, in accordance with accepted academic practice. No use, distribution or reproduction is permitted which does not comply with these terms. 